\title{
RABDOMIOSARCOMA BOTRIOIDE DEL APARATO GENITAL FEMENINO
}

\section{Botryoid rhabdomyosarcoma of the female genital tract}

\section{Dr. Álvaro M. Gartner Caballero*, Dra. Madeline F. Quezada Dalmau**}

Recibido: 10/3/2018 Aprobado: 14/4/2018

Cómo citar: Gartner-Caballero Á, Quezada-Dalmau M, Rabdomiosarcoma Botrioide del aparato genital femenino Ciencia y Salud [Internet]. 2018 mayo 1; [citado 2018 agosto 15]; 2(2):[aprox. 0 p.].

Disponible en: https://revistas.intec.edu.do/index.php/cisa/article/view/1183

\section{Resumen}

El rabdomiosarcoma del tracto genital inferior es una neoplasia maligna denominada heteróloga, por asentarse en localizaciones donde no existe músculo estriado. Las series publicadas coinciden en que la localización típica de este tumor genital en la infancia es la vagina, mientras que en la segunda y tercera décadas de la vida es el cérvix uterino $^{4,6}$.

La presentación clínica del rabdomiosarcoma embrionario variedad botrioides es, en general, una masa que protruye por la uretra o el introito vaginal, o por la presencia de flujo fétido o sangrado vaginal en niñas menores de 2 años. Se presenta el caso de un rabdomiosarcoma botrioides de la vagina diagnosticado en una paciente de 13 años y virgen. El apoyo diagnóstico con inmunohistoquímica es de vital importancia y la evaluación médica multidisciplinaria precoz y oportuna permitirá siempre establecer un diagnóstico y tratamiento adecuados que mejoren el pronóstico de quienes padecen esta enfermedad.

Palabras clave: Rabdomiosarcoma; botrioide; vagina; tumor; maligno.

\footnotetext{
* Ginecólogo Oncólogo. Instituto de Oncología Dr. Heriberto Pieter, República Domincana. amegartner@hotmail.com

** Ginecólogo Obstetra, Fellowship II Instituto de Oncología Dr. Heriberto Pieter, República Dominicana. franqueza6@ gmail.com
}

\section{Summary}

Rhabdomyosarcoma of the lower genital tract is a malignant neoplasm called heterologous, because it sits in locations where there is no striated muscle. The published series coincide in that the typical location of this genital tumor in childhood is the vagina, while in the second and third decades of life it is the uterine cervix ${ }^{4,6}$.

The clinical presentation of botryoid embryonic rhabdomyosarcoma variety is, in general, a mass that protrudes through the urethra or vaginal introitus, or the presence of fetid flow or vaginal bleeding in girls under 2 years. The case of a botoxides rhabdomyosarcoma of the vagina diagnosed is a 13-year-old and virgin patient. Diagnostic support with immunohistochemistry is of vital importance and early and timely multidisciplinary medical evaluation will always allow for an adequate diagnosis and treatment to improve the prognosis of those suffering from this disease.

Keywords: Rhabdomyosarcoma; botryoid; vagina; tumor; malignant 


\section{Introducción}

El rabdomiosarcoma infantil, es el tumor maligno de tejido blando más frecuente. Representa aproximadamente $3.5 \%$ de los casos de cáncer en niños de 0 a 14 años de edad. Según el Intergroup Rhabdomyosarcoma Study, la máxima incidencia del rabdomiosarcoma embrionario genital se registra en menores de catorce años, cuya ubicación típica es la vagina. La localización cervical, cuatro veces menos frecuente, es propia de adolescentes y adultas jóvenes ${ }^{10}$.

El rabdomiosarcoma fue descrito inicialmente por Webner, en 1854. Es un tumor complejo y de gran malignidad que se origina en las células del mesénquima embrionario con capacidad para diferenciarse en células musculares esqueléticas ${ }^{1}$.

Se han descrito 3 tipos histológicos de rabdomiosarcoma: embrionario (6 \%), alveolar (20\%) y pleomórfico $(1 \%)$. El primero, que se subdivide en las variedades botrioides y fusocelular, es el más frecuente en la niñez. Representa alrededor del 60$70 \%$ de los casos de rabdomiosarcoma infantiles y en ese grupo etario ${ }^{1,4}$.

La localización vaginal es rara, encontrándose frecuentemente en infantes femeninas menores de 5 años de edad ( $90 \%$ de los casos) y cerca de las dos terceras partes del total durante los primeros dos años de vida ${ }^{5}$.

La etiología de este sarcoma es desconocida. Es una neoplasia altamente maligna, de origen multifocal $^{1,4}$, que crece como masas voluminosas, polipoides, que pueden llenar y sobresalir de la vagina, con aspecto de racimo de uvas (botrioide) ${ }^{6}$. $\mathrm{Su}$ consistencia es gelatinosa, friable y desprende fragmentos, produciendo hemorragias vaginales e infecciones secundarias ${ }^{6}$.

El pronóstico de esta enfermedad está determinado por variables como el tamaño tumoral, el órgano comprometido, la edad del paciente, el resultado quirúrgico (R0/R1) y la presencia de metástasis ${ }^{13}$.

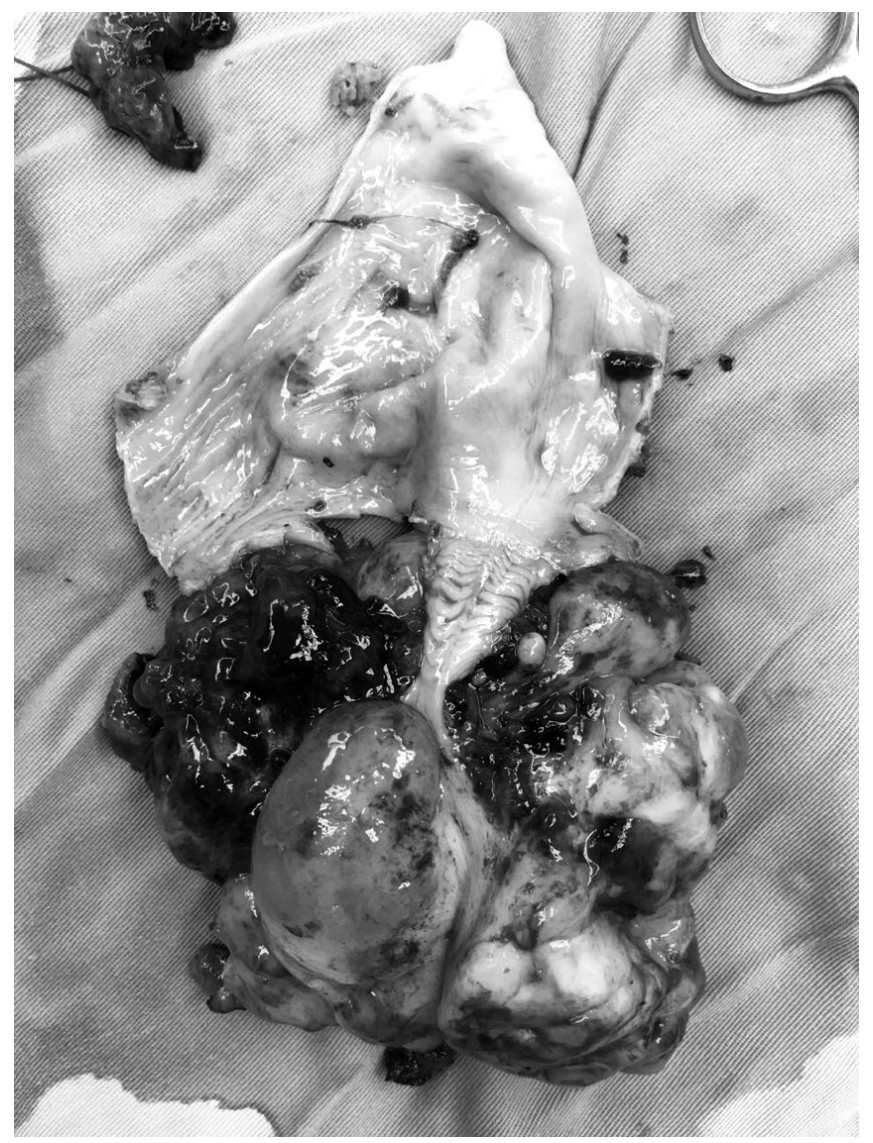

De esta manera, niños con tumores localizados en vía biliar, vagina y región de la cabeza y el cuello, menores de $5 \mathrm{~cm}$ de diámetro y que no presentan metástasis ganglionares y/o viscerales tendrían mejores tasas de supervivencia ${ }^{5,6}$.

La evaluación médica multidisciplinaria precoz y oportuna permitirá siempre establecer un diagnóstico y tratamiento adecuados que mejoren el pronóstico de quienes padecen esta enfermedad ${ }^{1}$. Por este motivo es imprescindible que a las consultas médicas especializadas en Ginecología infanto juvenil sean remitidas todas aquellas niñas en las que sus familiares o el propio médico observen cualquier anormalidad en su aparato genital.

Esta entidad, aunque es poco frecuente, puede presentarse en nuestro medio. Es por esto que perseguimos con este trabajo realizar la revisión del tema y la presentación de un caso típico de Sarcoma botrioide de vagina. 


\section{Método}

Se revisó la historia clínica de una paciente ingresada con el diagnóstico de Sarcoma botrioide de vagina en el año 2017.

Además, se confeccionó una ficha donde se recogieron los siguientes datos:

- Nombre de la paciente

- Edad, sexo, raza

- Motivo de ingreso

- Historia de la enfermedad actual

- Datos positivos al examen físico

- Resultados de los estudios complementarios

- Tratamiento indicado

\section{Presentación del caso clínico}

Paciente femenina de 13 ańos de edad, con antecedentes mórbidos negados. Conocida en la consulta de ginecología cuando acude referida desde otro centro de salud con reporte histopatológico de laparotomía exploratoria más biopsia por congelación de cuña de ambos ovarios por masa anéxial: para ovario derecho: tumor del estroma de los cordones sexuales, que incluye tumor de células de la granulosa tipo juvenil y tumor de células de Sertoly-Leydig; y en ovario izquierdo: quistes foliculares. Se realiza inmunohistoquímica que reporta tumor de células de Sertoly-Leydig. Trae reporte de resonancia magnética de pelvis, masa a nivel de vagina distal, que desplaza estructuras adyacentes sin invasión local probablemente neoformativa, ovario derecho con alteración de contorno y pobremente delimitado. Marcadores tumorales (CA-125, Alfafetoproteína, HE4) dentro de parámetros normales. Los estudios de laboratorio, la citometría hemática, la química sanguinea, las pruebas de funcionamiento hepático y los tiempos de coagulación fueron normales.

Con el diagnóstico ya mencionado se decide llevar a cirugía, donde se le realiza laparotomía exploratoria, toma de citología de líquido peritoneal, salpingooforectomía derecha, tumorectomía y omentectomía. En el transquirúrgico se evidencia una masa en la vagina que protruía a través del fondo de saco posterior de 10x12 cm., la cual se extirpa en su totalidad, no dejando lesión macroscópica visible.

El informe definitivo de patología mostró masa de vagina: sarcoma de bajo grado a favor de rabdomiosarcoma botrioide, tejido de ovario: tumor de células de Sertoly y Leydig de grado intermedio, omento: tejido fibroadiposo, citología de líquido peritoneal: negativo para malignidad.

Al mes de la cirugía, acude presentando dolor y distensión abdominal, por lo que se realiza TAC de abdomen y pelvis que reporta: gran masa pélvica, con componente sólido, con un componente superior que se adosa a la pared abdominal, pudiendo corresponder a implantes, sin que se descarte otra posibilidad, compresión de pared posterior de la vejiga y la pared anterior del recto.

Es llevada a cirugía nuevamente, donde se le realiza laparotomía exploratoria, evidenciando gran cantidad de tejido sarcomatoso. Se realiza citorreducción subóptima.

Al momento la paciente se encuentra en espera de inicio de protocolo de quimioterapia.

\section{Estudios de extensión:}

Sonografia abdominal (9/03/2017): valoramos en cavidad abdominal imagen de masa heterogénea, que muestra en su interior áreas anecoides con relación a degeneración, diámetros aproximados de $12.8 \times 8.0 \mathrm{~cm}$ y astitis.

Sonografía transvaginal (12/03/2017): lesión de masa anexial derecha, de $16.9 \times 16.7 \mathrm{~cm}$, líquido libre en cavidad pélvica.

Tomografía de abdomen (13/03/2017): lesión de masa compleja en cavidad abdomino-pélvica, a 
valorar lesión neoproliferativa, astitis, derramepleural bilateral.

Rx Tórax (16/03/2017): derrame pleural derecho.

\section{Marcadores tumorales}

Bhcg: $2 \mathrm{mU} / \mathrm{ml}$, CA 125: $148 \mathrm{U} / \mathrm{ml}$, Alfafetoproteína: $1.63 \mathrm{ng} / \mathrm{ml}$

\section{Discusión}

Los rabdomiosarcomas representan el 50\% de los sarcomas de tejidos blandos en menores de 15 ańos de edad; por tanto, es el sarcoma más común en la infancia con una incidencia de 4 a 7 niños por millón ${ }^{3,4}$. Corresponden al 5-8\% de todos los tipos de cáncer en ese grupo etario ${ }^{12}$, con una prevalencia ligeramente mayor en el sexo masculino (relación 3:2) ${ }^{1}$. Alrededor del 50\% de todos los rabdomiosarcomas ocurre en menores de 5 años de edad de ellos, solo un $2 \%$ está presente al momento del nacimiento ${ }^{1,3}$. La presentación cutánea primaria es extremadamente rara $(0.7 \%)^{2,4} \mathrm{y}$, aunque escasas, las metástasis cutáneas pueden ser la primera manifestación de la enfermedad ${ }^{4}$. El tumor se origina en el mesénquima embrionario, precursor del músculo estriado fetal en las semanas 7 a 10,4,5; por tal razón, son tumores derivados de los rabdomioblastos o las células musculares primitivas. Esto se ha confirmado porque expresan los marcadores tumorales miogenina y mio D1 característicos de este tipo de neoplasias ${ }^{4}$. Los rabdomiosarcomas pueden aparecer en diferentes partes del cuerpo, obedeciendo a la siguiente distribución, por su frecuencia: cabeza y cuello, 35-40\%; tracto genitourinario, 20\%; extremidades, $15-20 \%$; y tronco, pulmón o intraabdominal, $10-15 \%{ }^{1}$.

La mayoría de los rabdomiosarcomas se desarrollan de forma espontánea, sin que exista algún factor predisponente conocido, pero en ocasiones ocurren en asociación con otros trastornos como la neurofibromatosis, el Síndrome de Costello, el Síndrome de Beckwith-Wiedemann, el Síndrome de Li Fraumeni, o en individuos con antecedentes de cáncer mamario materno ${ }^{4,6}$.

La variedad botrioides (del griego botrys, racimo; eidos, aspecto) es la forma polipoide del rabdomiosarcoma embrionario. Se caracteriza por múltiples proyecciones polipoides que forman racimos de consistencia gelatinosa, friables y que se desprenden en fragmentos, ocasionando hemorragias a menudo. Los tumores de este tipo representan cerca de un $10 \%$ de todos los casos de rabdomiosarcoma y suelen ser tumores embrionarios que se desarollan bajo la superficie mucosa de orificios corporales como la vagina y la nariz; también se ha visto afectado el tracto biliar ${ }^{3}$.

Dado su origen muscular, el diagnóstico puede apoyarse en la inmunohistoquímica positiva para actina, miogenina, desmina y vimentina ${ }^{3}$, mas el diagnóstico histopatológico diferencial debe contemplar toda la variedad de tumores malignos de células redondas como Sarcoma de Ewing, tumor neuroectodérmico primitivo y linfoma, en los que los marcadores musculares son negativos ${ }^{7}$. La presentación clínica del rabdomiosarcoma embrionario en la variedad botrioides es, en general, una masa que protruye por la uretra o el introito vaginal, o por la presencia de flujo fétido o sangrado vaginal en niñas menores de 2 años $^{5,6}$. El pronóstico está determinado por variables como el tamaño del tumor, el sitio de origen, la edad del paciente, la enfermedad residual postquirúrgica y la presencia de metástasis al momento de establecer el diagnóstico.

Los sitios primarios de pronóstico más favorable incluyen órbita, paratesticulares, vagina y tracto bi$\operatorname{liar}^{14}$. El tamaño del tumor al momento del diagnóstico tiene relevancia pronóstica. Los pacientes con neoplasias de tamaño inferior a $5 \mathrm{~cm}$ tienen mejores expectativas de sobrevida que los individuos con tumores mayores de $5 \mathrm{~cm}$, en tanto que el pronóstico para los niños con enfermedad metastásica al momento del diagnóstico suele ser malo ${ }^{1}$. 
La magnitud de la enfermedad residual posterior a la cirugía inicial también guarda correlación con el pronóstico. Según el Intergrupo de Estudio para Rabdomiosarcoma, la sobrevida a 5 años en pacientes con enfermedad residual voluminosa (Grupo Clínico III) es de, aproximadamente, un 70\% contra $90 \%$ de sobrevida a 5 años en pacientes sin tumor residual postquirúrgico (Grupo clínico I) y un $80 \%$ de sobrevida a 5 ańos para pacientes con tumor residual microscópico después de la cirugía (Grupo clínico II) ${ }^{1}$. Se ha postulado que el 3-9\% de los tumores botrioides reinciden transcurridos unos 5 años de haber remitido la enfermedad, reduciendo la tasa de supervivencia a un $64 \%$. Las reincidencias suelen ocurrir, con mayor frecuencia (95\%), dentro de los 3 primeros ańos posteriores al diagnóstico y al tratamiento, y son más frecuentes en pacientes con diagnóstico de rabdomiosarcoma estado III o IV ${ }^{2,5}$.

El tratamiento se fundamenta en el estadio clínico, la localización del tumor primario y la extensión de la enfermedad. Se centra en conseguir un "control local" y un "control sistémico". El control local hace referencia a la erradicación permanente del "tumor primario". Esto se lleva a cabo habitualmente mediante extirpación quirúrgica o irradiación del tumor (o ambos) y de cualquier área cercana afectada, añadiendo además tratamiento quimioterápico ${ }^{9,11}$.

Todos los pacientes con rabdomiosarcoma requieren quimioterapia para aumentar al máximo las posibilidades de curación. La mayoría de los niños en los Estados Unidos son tratados siguiendo los preceptos de un ensayo clínico internacional inicialmente denominado Estudio Intergrupo del Rabdomiosarcoma (ahora conocido como Comité de sarcomas de Partes Blandas del Grupo de Oncología Pediátrica).

Los fármacos más comunmente utilizados en el tratamiento del RMS en Estados Unidos y en Europa son: Vincristina, Dactinomicina, Ciclofosfamida, Topotecan, Irinotecan, Etopósido, Ifosfamida, Doxorrubicina y Carboplatino ${ }^{15}$.

Las niñas con rabdomiosarcoma embrionario del tracto genital (vagina, vulva, cérvix y útero) para las que la cirugía conservadora inicial es la regla pueden con frecuencia ser controladas mediante biopsias seriadas, comenzando aproximadamente 12 semanas después de completado el tratamiento quimioterápico, sin radioterapia adicional, aunque en casos seleccionados (generalmente restringidos a nińos con RMS parameníngeo que ha erosionado la base craneal extendiéndose al interior de la bóveda), la radiación debe de comenzar al mismo tiempo que la quimioterapia (o tan cercana como sea posible).

Dependiendo de la localización, el tamaño y el grupo en que se incluya el tumor, se administran entre 20 y 28 sesiones de radioterapia ${ }^{8}$. De forma óptima, el tratamiento ha de planificarse sobre imágenes tridimensionales del tumor de forma previa a la toma de biopsia y al suministro de quimioterapia. Nunca será suficientemente enfatizada la importancia de la habilidad del oncólogo radioterapeuta en el éxito del tratamiento del RMS. Puesto que se trata de tumores poco frecuentes, y dado que la mayoría de los niños con RMS son tratados mediante protocolos en los que se especifica de forma detallada la terapia a seguir en cada caso, el oncólogo radioterapeuta debe de ser capaz no solo de interpretar con rigor los estudios de imagen relevantes para diseñar un "campo de tratamiento" apropiado, que abarque la totalidad del tumor original, añadiendo además un margen adicional de tejido circundante, sino también de llevarlo a cabo en el tiempo especificado en el protocolo, teniendo en cuenta la tolerancia del tejido normal en las estructuras sanas circundantes y los riesgos de complicaciones a largo plazo derivados de la irradiación de tejidos en crecimiento en niños pequeños. 


\section{Bibliografía}

1. Monroy Prado GA, Toledo Bahena ME, Valencia Herrera A, Ramírez Cortés E, Mena Cedillos C. Rabdomiosarcoma genitourinario variedad botrioides: informe de un caso. Dermatología CMQ. 2013 [citado 25 abr 2016];11(3):208-212.

2. Junco Gelpi DA, Blanco Trujillo F, Montoya Cardero E, Junco Anaya DM, Anaya Correoso SM Rabdomiosarcoma pleomórfico del muslo. MEDISAN. 2015 [citado 25 abr 2016];19(2):252.

3. Tapia O. Rabdomiosarcoma Embrionario Uterino. Aspectos Morfológicos e Inmunohistoquímicos. Int J Morphol 2011; [citado 25 abr 2016]; 29(4) 29: 1126-1129.

4. Figueroa Carbajal JJ, Cárdenas Cardós R, Rivera Luna R, Castellanos Toledo A. Rabdomiosarcoma, experiencia de siete años en el Instituto Nacional de Pediatría. GAMO [Internet]. 2010 [citado 25 abr 2016];9(5):198-207.

5. Oscar Tapia E. Rabdomiosarcoma Embrionario Uterino. Aspectos Morfológicos e Inmunohistoquímicos. Int. J. Morphol [Internet]. 2011 [citado 25 abr 2016];29(4):1126-1129. Disponible en: http://www.scielo.cl/scielo. php?pid=S071795022011000400009\&scrip$\mathrm{t}=$ sci_arttext

6. Walterhouse DO, Meza JL, Breneman JC, Donaldson SS, Hayes-Jordan A et al. Local control and outcome in children with localized vaginal rhabdomyosarcoma: a report from the Soft Tissue Sarcoma Committee of the Children's Oncology Group. Pediatr Blood Cancer [Internet] 2011 [citado 25 abr 2016];57(1):76-83. Disponible en: http://www.ncbi.nlm.nih.gov/pmc/ articles/PMC3459820/

7. Wharam MD, Meza J, Anderson J, et al. Failure pattern and factors predictive of local failure in rhabdomyosarcoma: A report of Group III patients on the Third Intergroup Rhabdomyosarcoma Study. Journal of Clinical Oncology 2004; 22 (4):1902-1908.

8. Schuck A, Mattke AC, Schmidt B, et al. Group II rhabdomyosarcoma and rhabdomyosarcomalike tumors: Is radiotherapy necessary? Journal of Clinical Oncology. 2004;22 (3):143-149.

9. Raney RB, Stoner JA, Walterhouse DO et al. Results of treatment of fifty-six patients with localized retroperitoneal and pelvic rhabdomyosarcoma: A report from the Intergroup Rhabdomyosarcoma Study-IV, 1991-1997. Pediatric Blood and Cancer. 2004; 42 (2): $1-8$.

10. Houghton JP, McCluggage WG. Embryonal rhabdomyosarcoma of the cervix with focal pleomorphic areas. J Clin Pathol. 2007;60 (5):88-9.

11. Maharaj NR, Nimako D, Hadley GP. Multimodal therapy for the initial management of genital embryonal rhabdomyosarcoma in childhood. Int J Gynecol Cancer. 2008;18 (2):190-2.

12. Labaran Dayyabu A, Adogu IO, Makama BS. Sarcoma botryoides a management dilemma: A review of two cases. Int J Case Rep Images [Internet]. 2014 [citado 25 abr 2016];5(7):482487. Disponible en: http:// www.ijcasereportsandimages.com/archive/2014/007-2014-ijcri/CS-10044-07-2014dayyabu/ijcri-1004407201444-dayyabu-full-text.php.

13. Pantoja Ludueńa M, Riveros Moron A, Salvatierra Frontanilla I, Parra Nigañez P. Rabdomiosarcoma botrioide de vagina. Rev. bol. ped [Internet]. 2012 [citado 25 abr 2016]; 15(3): [Aprox.4p]. Disponible en: http://www.scielo. 
org.bo/scielo.php?script=sci_arttext\&pi$\mathrm{d}=$ S1024-06752012000300007.

14. Junco Gelpi DA, Blanco Trujillo F, Montoya Cardero E, Junco Anaya DM, Anaya Correoso SM. Rabdomiosarcoma pleomórfico del muslo. MEDISAN. 2015 [citado 25 abr 2016];19(2):252. Disponible en: http://scielo.sld.cu/scielo.php?pi$\mathrm{d}=$ S102930192015000200014\&script $=$ sci_ arttext\&tlng=en.

15. Instituto Nacional del Cáncer. Disponible en: http://www.cancer.gov/espanol. 